\title{
Room-Temperature Quantum Bit Memory Exceeding One Second
}

\section{The Harvard community has made this article openly available. Please share how this access benefits you. Your story matters}

\begin{tabular}{|l|l|}
\hline Citation & $\begin{array}{l}\text { Maurer, Peter Christian, Georg Kucsko, C. Latta, L. Jiang, . Room- } \\
\text { Norman Ying Yao, S. D. Bennett, F. Pastawski, et al. 2012. "Rom } \\
\text { Temperature Quantum Bit Memory Exceeding One Second." Science } \\
336 \text { (6086) (June 8): 1283-1286. }\end{array}$ \\
\hline Published Version & doi:10.1126/science.1220513 \\
\hline Citable link & http://nrs.harvard.edu/urn-3:HUL.InstRepos:12132060 \\
\hline Terms of Use & $\begin{array}{l}\text { This article was downloaded from Harvard University's DASH } \\
\text { repository, and is made available under the terms and conditions } \\
\text { applicable to Open Access Policy Articles, as set forth at http:// } \\
\text { nrs.harvard.edu/urn-3:HUL.InstRepos:dash.current.terms-of- } \\
\text { use\#OAP }\end{array}$ \\
\hline
\end{tabular}




\title{
Room temperature quantum bit memory exceeding one second
}

\author{
P. C. Maurer ${ }^{\dagger}{ }^{1}$ G. Kucsko ${ }^{\dagger},{ }^{1}$ C. Latta,${ }^{1}$ L. Jiang, ${ }^{2}$ N. Y. Yao, ${ }^{1}$ \\ S. D. Bennett, ${ }^{1}$ F. Pastawski, ${ }^{3}$ D. Hunger,${ }^{3}$ N. Chisholm,${ }^{4}$ M. \\ Markham, ${ }^{5}$ D. J. Twitchen, ${ }^{5}$ J. I. Cirac, ${ }^{3}$ and M. D. Lukin ${ }^{* 1}$ \\ ${ }^{1}$ Department of Physics, Harvard University, Cambridge, MA 02138, USA \\ ${ }^{2}$ Institute for Quantum Information and Matter, \\ California Institute of Technology, Pasadena, California 91125, USA \\ ${ }^{3}$ Max-Planck-Institut für Quantenoptik, Garching, D-85748, Germany \\ ${ }^{4}$ School of Engineering and Applied Sciences, \\ Harvard University, Cambridge, MA 02138, USA \\ ${ }^{5}$ Element Six Ltd, Ascot SL5 8BP, UK
}

\begin{abstract}
Stable quantum bits, capable of both storing quantum information for macroscopic timescales and of integration inside small, portable devices, represent an essential building block for an array of potential applications. We demonstrate high fidelity readout of a solid-state qubit, which preserves its polarization for several minutes and features coherence lifetimes exceeding one second at room temperature. The qubit consists of a single ${ }^{13} \mathrm{C}$ nuclear spin in the vicinity of a Nitrogen-Vacancy (NV) color center within an isotopically purified diamond crystal. The long qubit memory time is achieved via a novel technique involving dissipative decoupling of the single nuclear spin from its local environment. The versatility, robustness and potential scalability of this system may allow for new applications in quantum information science.
\end{abstract}

Many applications in quantum communication [1] and quantum computation [2] rely upon the ability to maintain qubit coherence for extended periods of time. Furthermore, integrating such quantum mechanical systems in compact, mobile devices remains an outstanding experimental task. While trapped ions and atoms [3] can exhibit coherence times as long as minutes, they typically require a complex infrastructure involving laser-cooling and ultra-high vacuum. Other systems, most notably ensembles of electronic and nuclear 
spins, have also achieved long coherence times in bulk ESR and NMR experiments [4-6]; however, owing to their exceptional isolation, individual preparation, addressing and high fidelity measurement remains challenging, even at cryogenic temperatures [7].

Our approach is based upon an individual nuclear spin in at room-temperature solid. A nearby electronic spin is used to initialize the nuclear spin [8-10] in a well defined state and to read it out in a single shot [10] with high fidelity. A combination of laser illumination and RF decoupling pulse sequences $[4,11]$ enables the extension of our qubit memory lifetime by nearly three orders of magnitude. This approach decouples the nuclear qubit from both the nearby electronic spin and other nuclear spins, demonstrating that dissipative decoupling can be a robust and effective tool for protecting coherence in various quantum information systems $[2,12,13]$.

Our experiments utilize an individual NV center and a single ${ }^{13} \mathrm{C}(I=1 / 2)$ nuclear spin (Fig. 1A) in a diamond crystal. We work with an isotopically pure diamond sample, grown using Chemical Vapor Deposition from isotopically enriched carbon consisting of $99.99 \%$ spinless ${ }^{12} \mathrm{C}$ isotope. In such a sample, the optically detected electron spin resonance (ESR) associated with a single NV center is only weakly perturbed by ${ }^{13} \mathrm{C}$ nuclear spins, resulting in long electronic spin coherence times [14]. This allows us to make use of a Ramsey pulse sequence to detect a weakly coupled single nuclear spin, separated from the NV by 1-2 nanometers. The coupling strength at such a distance is sufficient to prepare and measure the nuclear spin qubit with high fidelity. For the present concentration of ${ }^{13} \mathrm{C}$ nuclei, about $10 \%$ of all NV centers exhibit a coupled nuclear spin with a separation of this order.

In our experimental setup, the diamond sample is magnetically shielded from external perturbations, and a static magnetic field $B=(244.42 \pm 0.02) \mathrm{G}$ is applied along the NV symmetry axis. The spin transition between the $|0\rangle \rightarrow|-1\rangle$ electronic spin states is addressed via microwave radiation [15]. Figure 1B shows the free electron precession of an individual NV center, measured via a Ramsey sequence. The signal dephases on a time scale of $T_{2 e}^{*}=(470 \pm 100) \mu s$ consistent with the given isotopic purity of the sample [14]. The characteristic collapses and revivals of the Ramsey signal correspond to the signature of a single weakly coupled ${ }^{13} \mathrm{C}$ nuclear spin. This coupling strength, originating from a hyperfine interaction, corresponds to an electron-nuclear separation of roughly $1.7 \mathrm{~nm}$ [15].

To confirm that the signal originates from a ${ }^{13} \mathrm{C}$ nuclear spin, we measure the probability of a RF-induced nuclear spin-flip as a function of carrier frequency, $\omega$. As described below, 
we prepare the nuclear spin in either the $|\downarrow\rangle$ or $|\uparrow\rangle$-state by performing a projective measurement. After preparation of the nuclear spin via projection a 1.25 ms Gaussian shaped RF $\pi$-pulse is applied. A second step of nuclear measurement then allows us the nuclear spin-flip to be determined. Figure 1C shows that this probability is characterized by three resonances located at $\omega /(2 \pi)=258.86,261.52,264.18 \mathrm{kHz}$, corresponding to the NV electronic spin being in $m_{s}=1,0,-1$ respectively; this indicates a projected hyperfine interaction, $A_{\|}=(2 \pi)(2.66 \pm 0.08) \mathrm{kHz}$.

An important facet of quantum control involves the ability to perform high fidelity initialization and readout. We use repetitive readout to achieve single shot detection of the nuclear spin state. In this approach (Fig. 2A) the electronic spin is first polarized into the $|0\rangle$ state. Next, a $\mathrm{C}_{n} \mathrm{NOT}_{e}$ logic gate (electronic spin-flip conditioned on the nuclear spin) is performed and the resulting state of the electronic spin is optically detected; this sequence is repeated multiple times to improve the readout fidelity. The required quantum logic is achieved via a Ramsey sequence on the electronic spin, where the free precession time is chosen to be $\tau=\pi / A_{\|}$. Fig. 2B depicts an example trace of the accumulated fluorescence of 20000 readout repetitions per data point. The resulting signal clearly switches between two distinct values, which correspond to the two states of the spin- $\frac{1}{2}{ }^{13} \mathrm{C}$ nuclear spin. We associate high (low) count rates with the $|\uparrow(\downarrow)\rangle$ states of the nuclear spin, noting that these do not necessarily correspond to alignment/anti-alignment with the external field [15]. This time trace indicates that the nuclear spin preserves its orientation, on average, for about half a minute.

To achieve high fidelity initialization of the nuclear spin, we post-select repetitive readout measurements that are below (above) a threshold corresponding to 147 (195) counts per 2.2 s. This allows us to prepare the nuclear spin state with $>97 \%$ fidelity [15]. After successful initialization via projection, a second repetitive readout measurement is performed. This allows us to extract readout count statistics dependent on the nuclear spin state. As shown in Fig. 2C, the two distributions for the count rates of $|\uparrow\rangle$ and $|\downarrow\rangle$ are clearly resolved and their medians match the high and low levels of the fluorescence trace in Fig. 2B. From the overlap between the two distributions, we obtain a projective readout fidelity of (91.9 \pm 2.5$) \%$ [16].

The long spin orientation lifetime, extracted from Fig. $2 \mathrm{~B}$, implies that our ${ }^{13} \mathrm{C}$ nuclear spin is an exceptionally robust degree of freedom. To quantify the nuclear depolarization rate, the $T_{1 n}$ time was measured as a function of laser intensity. In the dark, no decay was 
observed on a timescale of $200 \mathrm{~s}$ [15]. However, consistent with predictions from a spinfluctuator model[17, 18], when illuminated with a weak optical field, $T_{1 n}$ drops to $(1.7 \pm 0.5) \mathrm{s}$ and increases linearly for higher laser intensities (Fig. 2D).

To probe the qubit's coherence time, our nuclear spin is again prepared via a projective measurement, after which, an NMR Ramsey pulse sequence is applied. The final state of the nuclear spin is then detected via repetitive readout. The results (Fig. 3B) demonstrate that, in the dark, the nuclear coherence time $T_{2 n}^{*}$ is limited to about $(8.2 \pm 1.3)$ ms. The origin of this relatively fast dephasing time can be understood by noting its direct correspondence with the population lifetime of the electronic spin $T_{1 e}=(7.5 \pm 0.8) \mathrm{ms}$ (blue curve Fig. 3B) [19]. Because the electron-nuclear coupling $A_{\|}$exceeds $1 / T_{1 e}$, a single (random) flip of the electronic spin (from $|0\rangle$ to $| \pm 1\rangle$ ) is sufficient to dephase the nuclear spin.

To extend the nuclear memory time, we must effectively decouple the electronic and nuclear spin during the storage interval. This is achieved by subjecting the electronic spin to controlled dissipation. Specifically, the NV center is excited by a focused green laser beam, resulting in optical pumping of the NV center out of the magnetic states $(| \pm 1\rangle)$. In addition, the NV center also undergoes rapid ionization and deionization at a rate $\gamma$, proportional to the laser intensity. When these transition rates exceed the hyperfine coupling strength, the interaction between the nuclear and electronic spin is strongly suppressed owing to a phenomenon analogous to motional averaging [17].

Using this decoupling scheme, we show in Fig. 3C that the nuclear coherence time can be enhanced by simply applying green laser light; in particular, $10 \mathrm{~mW}$ of green laser excitation, yield an extended nuclear coherence time of $T_{2 n}^{*}=(0.53 \pm 0.14) \mathrm{s}$. An improvement of $T_{2 n}^{*}$ by almost two orders of magnitude compared with measurements in the dark. The dependence of $T_{2 n}^{*}$ on green laser intensity shows a linear increase for low intensities and saturates around one second (Fig. 3D).

The observed limitation of coherence enhancement arises from dipole-dipole interactions of the nuclear qubit with other ${ }^{13} \mathrm{C}$ nuclei in the environment. In our sample, we estimate this average dipole-dipole interaction to be $\sim 1 \mathrm{~Hz}$, consistent with the observed coherence time. Further improvement of the nuclear coherence is achieved via a homonuclear RFdecoupling sequence. The composite sequence (Fig. 3D) is designed to both average out the inter-nuclear dipole-dipole interactions (to first order) and to compensate for magnetic field drifts. Applying this decoupling sequence in combination with green excitation can further 
extend the coherence time to beyond one second (Fig. 3E, blue points).

These measurements demonstrate that individual nuclear spins in isotopically pure diamond represent an exceptional candidate for long-lived memory qubits. The qubit memory performance is fully quantified by two additional measurements. First, the average fidelity is determined by preparing and measuring the qubit along three orthogonal directions. This fidelity, $\bar{F}=\frac{1}{2}(1+\langle C\rangle)$, is extracted from the observed contrast $(C)$ of the Ramsey signal and is presented in Fig. 4A for two cases (with and without homonuclear decoupling) [8]. Even for memory times up to $(2.11 \pm 0.3) \mathrm{s}$, the fidelity remains above the classical limit of $2 / 3$. Finally, a full characterization of our memory (at one second of storage time) is obtained via quantum process tomography. The corresponding $\chi$-matrix (Fig. 4C) reveals an average fidelity of $\bar{F}=(87 \pm 5) \%$ [15].

To quantitatively understand the coherence extension under green illumination, we consider depolarization and dephasing of the nuclear spin due to optical illumination and interaction with the nuclear spin environment. Excitation with $532 \mathrm{~nm}$ (de)ionizes the NV center with a rate proportional to the laser intensity[20]. Adding up the peak probabilities (Fig. 1C) for the nuclear RF transitions reveals a total transition probability of $(63 \pm 5) \%$. This is consistent with recent observations, where, under strong green illumination, the NV center is found to spend $30 \%$ of its time in an ionized state [20]. In this state, RF induced nuclear transitions are suppressed since the depolarization rate of the electronic spin is much faster than the nuclear Rabi frequency [20]. Because the hyperfine interaction is much smaller than the electronic Zeemann splitting, flip-flop interactions between the electronic and nuclear spin can be neglected. However, in the presence of an off-axis dipolar hyperfine field $A_{\perp}$, nuclear depolarization still occurs at a rate ${ }^{1} / T_{1 n} \sim \frac{A_{\perp}^{2}}{\left(\gamma_{13} C^{B} / 2\right)^{2}+(\gamma)^{2}} \gamma[15]$. While this simple analysis is already in good agreement with our observations (Fig. 2D), further insight is provided by a detailed 11-level model of $\mathrm{NV}$ dynamics [15]. As $T_{1 n}$ limits our readout, a careful alignment of the external field (i.e. choosing $A_{\perp} \rightarrow 0$ ) and enhanced collection efficiency should enable readout fidelities greater than $99 \%$.

For (de)ionization rates $\gamma$ much larger than the hyperfine interaction, the dephasing rate depends on the parallel component of the dipole field, ${ }^{1} / T_{2 n}^{*}=\Gamma_{o p t}+\Gamma_{d d}$, where $\Gamma_{d d}$ is the spin-bath induced dephasing rate and $\Gamma_{\text {opt }} \sim \frac{A_{\|}^{2}}{\gamma}$ is the optically induced decoherence. The dashed red line in Fig. 3E demonstrates that this model is in good agreement with our data. Application of our decoupling sequence also allows us to suppress nuclear-nuclear 
dephasing. We find that the main imperfection in this decoupling procedure originates from a finite RF detuning [15]. Accounting for this imperfection, we find excellent agreement with our data, as shown by the dashed blue line in Fig. 3E. Moreover, this model indicates that the coherence time increases almost linearly as a function of applied laser intensity, suggesting a large potential for improvement.

The use of even higher laser intensities is limited by heating of the diamond sample, which causes drifts in the ESR transition [21]. However, this can be overcome via a combination of temperature control and careful transition-frequency tracking, yielding an order of magnitude improvement in the coherence time to approximately one minute. Further improvement can be achieved by decreasing the hyperfine and the nuclear-nuclear interaction strength through a reduction of the ${ }^{13} \mathrm{C}$ concentration, potentially resulting in hour-long storage times [15]. Finally, it is possible to use coherent decoupling sequences and techniques based upon optimal control theory [22], which scale more favorably than our current dissipation-based method. With such techniques, we estimate that the memory lifetime can approach the timescale of phonon-induced nuclear depolarization, measured to exceed $T_{1 n}^{\max } \sim 36 \mathrm{~h}[23]$.

As a future application of our techniques the realization of fraud resistant quantum tokens can be considered. Here, secure bits of information are encoded into long-lived quantum memories. Along with a classical serial number, an array of such memories, may possible constitutes a unique unforgeable token $[24,25]$. With a further enhancement of storage times, such tokens may potentially be used as quantum-protected credit cards or as quantum identification cards [25] with absolute security. Furthermore, NV-based quantum registers can take advantage of the nuclear spin for storage, while utilizing the electronic spin for quantum gates and readout $[8,9]$. In particular, recent progress in the deterministic creation of arrays of NV centers [27] and NV-C pairs'[28], enables the exploration of scalable architectures [26, 29]. Finally, recent experiments have also demonstrated the entanglement of a photon with the electronic spin-state of an NV center [30]. Combining the advantages of an ultra-long nuclear quantum memory with the possibility of photonic entanglement opens up novel routes to long-distance quantum communication and solid state quantum repeaters [1].

†These authors contributed equally. All authors contributed extensively to this work. 
*To whom correspondence should be addressed. E-mail: lukin@physics.harvard.edu.

[1] L. M. Duan, C. Monroe, Rev. Mod. Phys. 82, 1209 (2010)

[2] T. D. Ladd, et. al. Nature 464, 45-53 (2010)

[3] C. Langer. et al. Phys. Rev. Lett. 95, 060502 (2005)

[4] T. D. Ladd, D. Maryenko, Y. Yamamoto, Phys. Rev. B 71, 014401 (2005)

[5] M. V. Balabas, T. Karaulanov, M. P. Ledbetter, D. Budker, Phys. Rev. Lett. 105, 070801 (2010)

[6] A. M. Tyryshkin. et al. Nat. Materials 11, 143-147 (2012)

[7] A. Morello, et al. Nature 467, 687691 (2010)

[8] M. V. Gurudev, et al. Science 1, 1312-1316 (2007)

[9] T. van der Sar, et. al. http://arxiv.org/abs/arXiv:1202.4379v1 (2012)

[10] P. Neumann, et al. Science 329, 542-544 (2010)

[11] G. de Lange, Z. H. Wang, D. Rist, V. V. Dobrovitski, R. Hanson, Science 330, 60-63 (2010)

[12] J. T. Barreiro, et. al. Nature 470, 486-491 (2011)

[13] H. Krauter, et al. Phys. Rev. Lett. 107, 080503 (2011)

[14] G. Balasubramanian, et al. Nat. Mat. 8, 383-387 (2009)

[15] Materials and methods are available as supporting material on Science Online.

[16] A. H. Burrell, D. J. Szwer, S. C. Webster, D. M. Lucas, Phys. Rev. A 81, 040302 (2010)

[17] L. Jiang, et al. Phys. Rev. Lett. 100, 073001 (2008)

[18] L. Jiang, et al. Science 326, 267-272 (2009)

[19] P. Neumann et al. Science 6, 1326-1329 (2008)

[20] G. Waldherr, et al. Phys. Rev. Lett. 106, 157601 (2011)

[21] V. M. Acosta, et al. PRL 104, 070801 (2010)

[22] N. Khaneja, R. Brockett, R. J. Glaser, Phys. Rev. A 63, 032308 (2001)

[23] C. Terblanche, E. Reynhardt, J. van Wyk, Solid State Nuc. Mag. 20, 1-22 (2001)

[24] S. Wiesner, Sigact News 15, 78 (1983)

[25] F. Pastawski, N. Y. Yao, L. Jiang, M. D. Lukin, J.I. Cirac, http://arxiv.org/abs/1112.5456v1 (2011)

[26] N. Y. Yao, et al. http://arxiv.org/abs/1012.2864 
[27] D. M. Toyli, C. D. Weis, G. D. Fuchs, T. Schenkel, D. D. Awschalom, Nano Lett. 10(8), $31683172(2010)$

[28] P. Spinicelli, et al. New J. Phys. 13, 025014 (2011)

[29] P. Neumann, et al. Nature Physics 6, 249-253 (2010)

[30] E. Togan, et al. Nature 466, 730-734 (2010)

Acknowledgments: We thank F. Jelezko, P. Neumann, J. Wrachtrup, R. Walsworth, A. Zibrov and P. Hemmer for stimulating discussions and experimental help. Harvard University has applied to patent the dissipative decoupling scheme. This work was supported in part by the NSF, the Center for Ultracold Atoms, DARPA (QUEST program), Element 6, Packard Foundation, EU (DIAMANT program), the Fulbright Science and Technology Award (P.C.M.), the Swiss National Science Foundation (C.L.), Sherman Fairchild Foundation and the NBRPC (973 program) 2011CBA00300 (2011CBA00301) (L.J.).

\section{Supporting Online Material}

www.sciencemag.org/cgi/content/full/science.1216821/DC1

Materials and Methods

SOM Text

Figs. S1 to S12 
FIG.1: Experimental System. (A) The NV center with a proximal ${ }^{13} \mathrm{C}$ spin can be modeled as a simple four-level system to understand readout dynamics. Nuclear spin sublevels $|\uparrow\rangle$ and $|\downarrow\rangle$ are split by a Zeeman shift $\left(\gamma_{13 C} B\right)$ and addressed via RF radiation with Rabi frequency $\Omega_{R F}$. The electronic transition $|0\rangle \rightarrow|-1\rangle$ (red arrows) can be simultaneously driven by a microwave field with relative detuning given by the hyperfine coupling strength, $A=\sqrt{A_{\|}^{2}+A_{\perp}^{2}}$. (B) An electron Ramsey measurement as a function of free evolution time $(\mathrm{t})$, depicts beating due to the different hyperfine transitions and a $T_{2 e}^{*}=(470 \pm 100) \mu \mathrm{s}$. (C) NMR spectra of ${ }^{13} \mathrm{C}$, obtained via the depicted pulse sequence, demonstrate three different nuclear transitions corresponding to electronic spin states $m_{s}=0, \pm 1$. The pulse sequence contains a blue Gaussian RF pulse and two repetitive readouts (purple), $c_{1}$ and $c_{2}$.

FIG. 2: Qubit readout. (A) Circuit diagram of repetitive readout of the nuclear spin $|n\rangle$. The readout uses a $C_{n} N O T_{e}$ gate consisting of multiple repetitions of an electronic spin Ramsey and subsequent repolarization. (B) Fluorescence time trace showing single shot readout of the nuclear spin and corresponding quantum jumps. The integration time for a single point is $4.4 \mathrm{~s}$. (C) Histogram of continuous repetitive readouts (per $4.4 \mathrm{~s}$ ) showing two overlapping distributions of nuclear spin states: $|\downarrow\rangle$ (blue) and $|\uparrow\rangle$ (red) [15]. (D) Nuclear spin orientation lifetime, $T_{1 n}$ (here and below error bars are one standard deviation), as a function of laser power in the presence of illumination by a $532 \mathrm{~nm}$ laser. As shown in the inset, each data point is extracted from a series of two repetitive readout sequences. The solid red curve represents the theoretical prediction from the simple model of nuclear depolarization induced by the off-axis dipolar hyperfine field.

FIG. 3: Nuclear spin coherence. (A) Model for repolarization and ionization dynamics. In the $\mathrm{NV}^{-}$charge state, the electronic spin can be pumped via green illumination to $m_{s}=0$ at a rate $R$. (B) Nuclear Ramsey experiment (red curve) depicting a dephasing time $T_{2 n}^{*}=(8.2 \pm 1.3) \mathrm{ms}$. The origin of this dephasing is the depolarization of the electronic spin (blue curve), with $T_{1 e}=(7.5 \pm 0.8) \mathrm{ms}$. (C) Nuclear Ramsey experiment with concurrent green illumination, showing $T_{2 n}^{*}=(0.53 \pm 0.14) \mathrm{s}$. (D) Experimental sequence used to measure the nuclear coherence time. A modified Mansfield Rhim Elleman Vaughan (MREV) decoupling sequence [4] is utilized. It consists of 16 MREV-8 pulse trains 
interwoven with 8 phase-refocusing $\pi$-pulses. Each MREV-8 pulse sequence can be achieved through $\pi / 2$ rotations around four different axes. (E) Nuclear coherence as a function of green laser power. Red data constitute a measurement of $T_{2 n}$ using a nuclear spin echo; blue data $T_{2 n}$ contain the additional MREV sequence. The dashed fits are calculated from the spin-fluctuator model [15]. Each data point is extracted via a measurement analogous to C).

FIG. 4: Nuclear memory fidelity. (A) Average fidelity as a function of time obtained from states prepared along $|x\rangle=\frac{1}{\sqrt{2}}(|\downarrow\rangle+|\uparrow\rangle),|y\rangle=\frac{1}{\sqrt{2}}(|\downarrow\rangle+i|\uparrow\rangle)$ and $|z\rangle=|\downarrow\rangle$. The nuclear echo (red curve) is obtained at $10 \mathrm{~mW}$ of green power while the MREV sequence (blue curve) is obtained at $30 \mathrm{~mW}$ of green power. The square data point represents the fidelity extracted from process tomography. (B) Pulse sequence depicting the initialization of four different nuclear states and three subsequent rotations. (C) The $\chi$ matrix of the full quantum process tomography at one second of storage time with $30 \mathrm{~mW}$ of continuous green illumination [15]. 
Figure 1

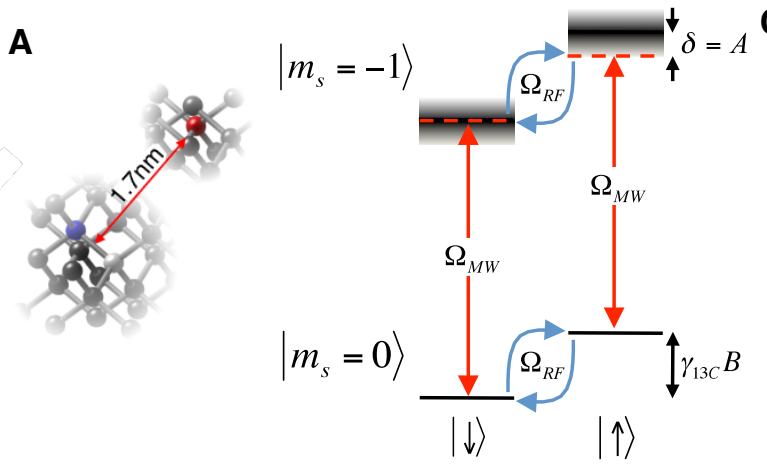

B
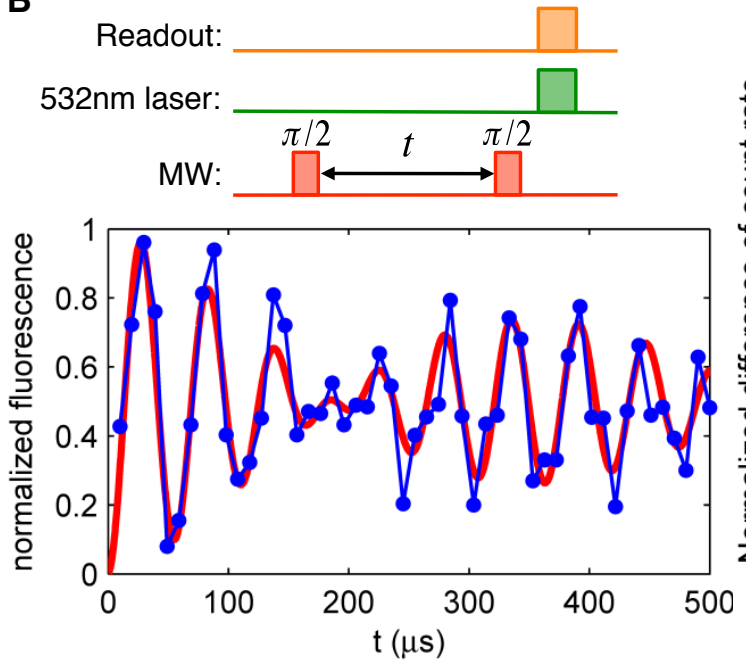

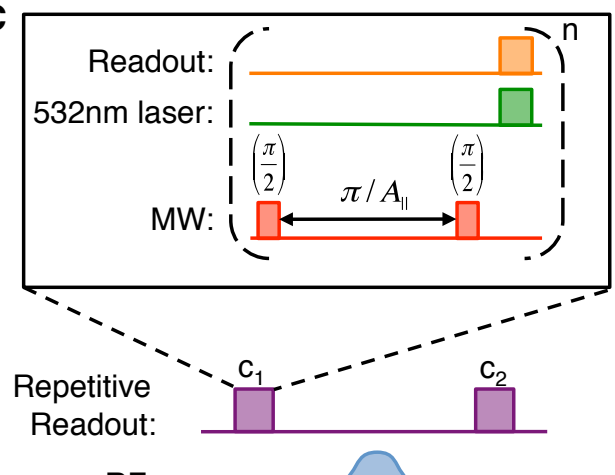

RF:

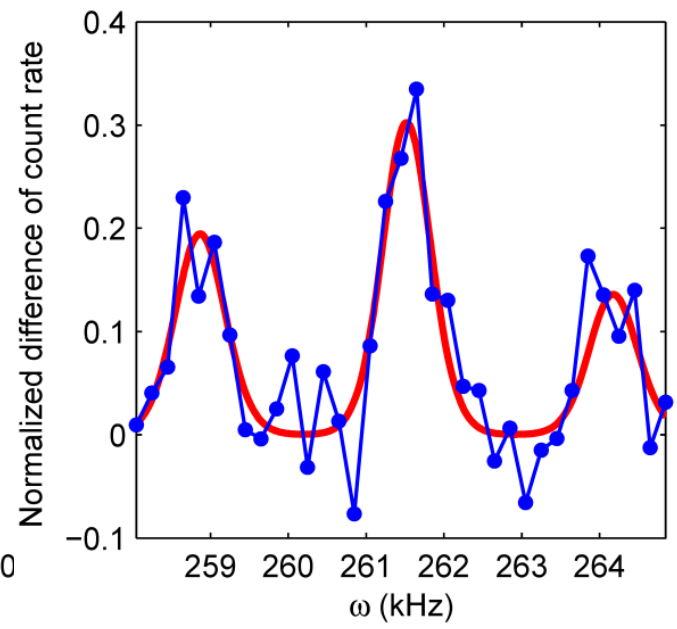




\section{Figure 2}

A

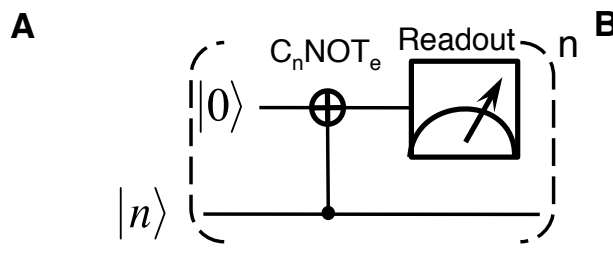
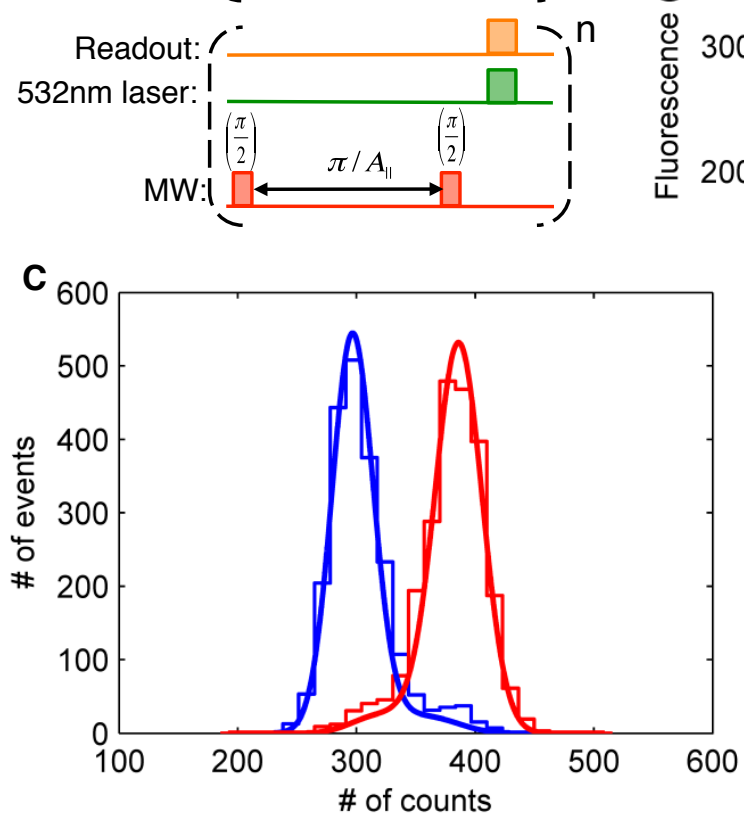

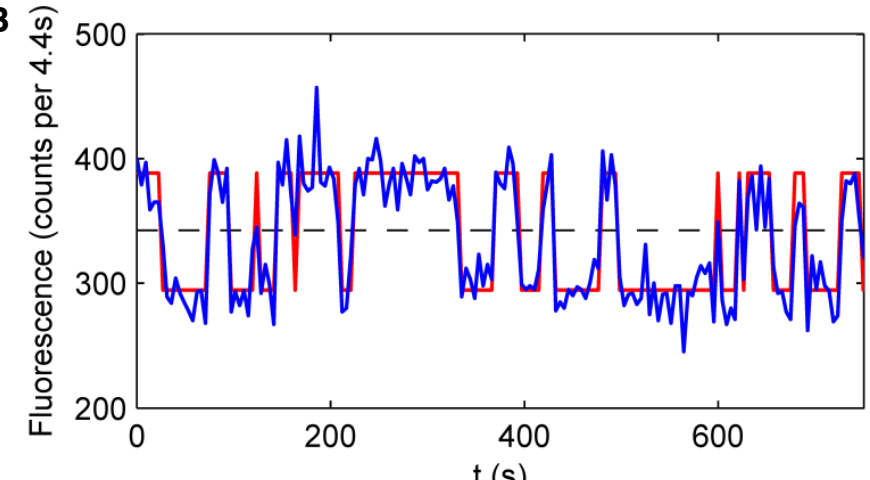

D

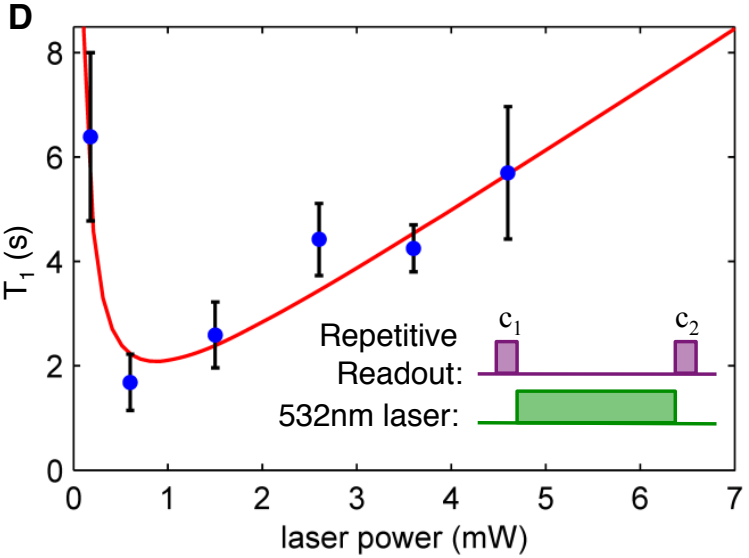


Figure 3

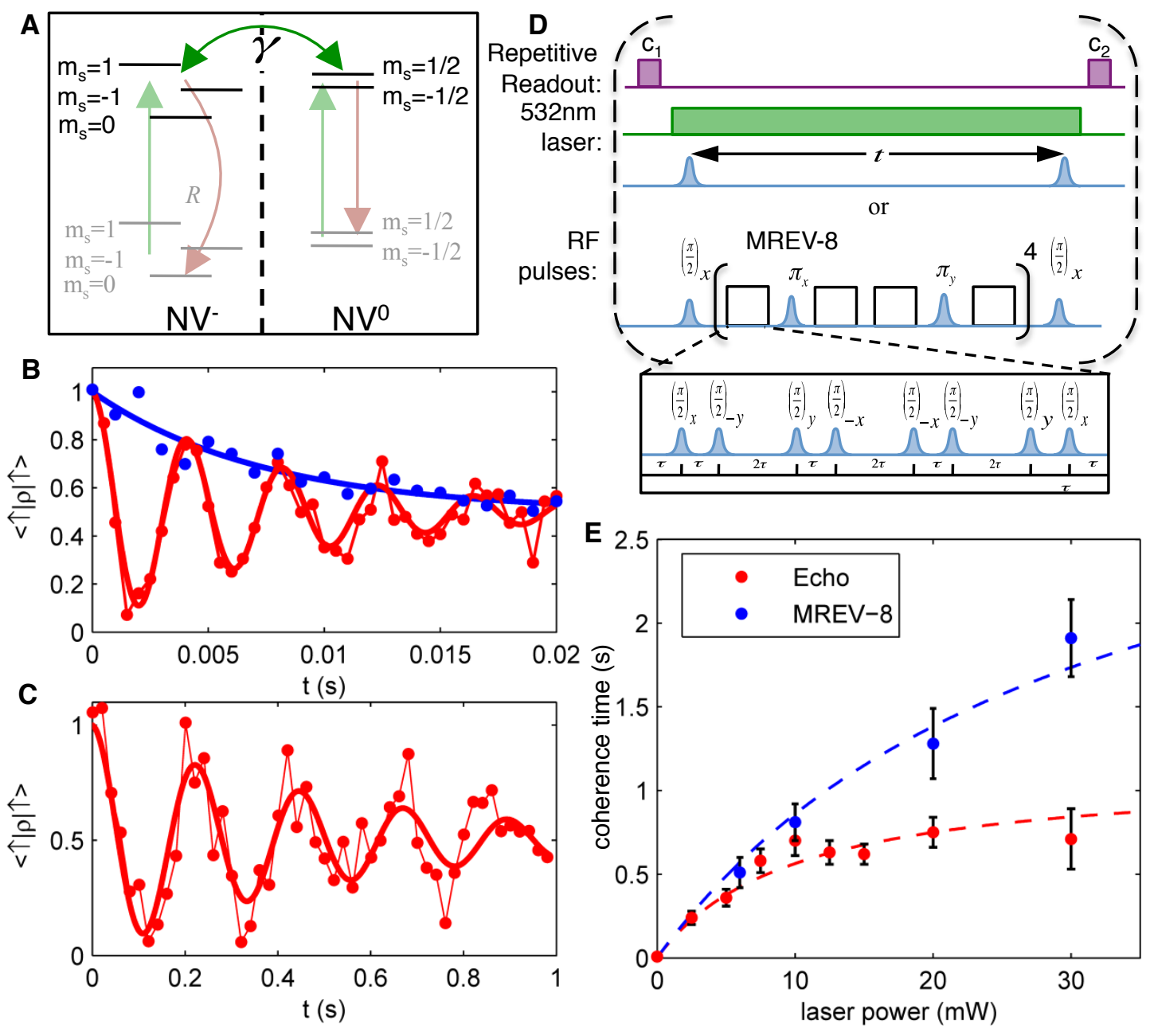


Figure 4
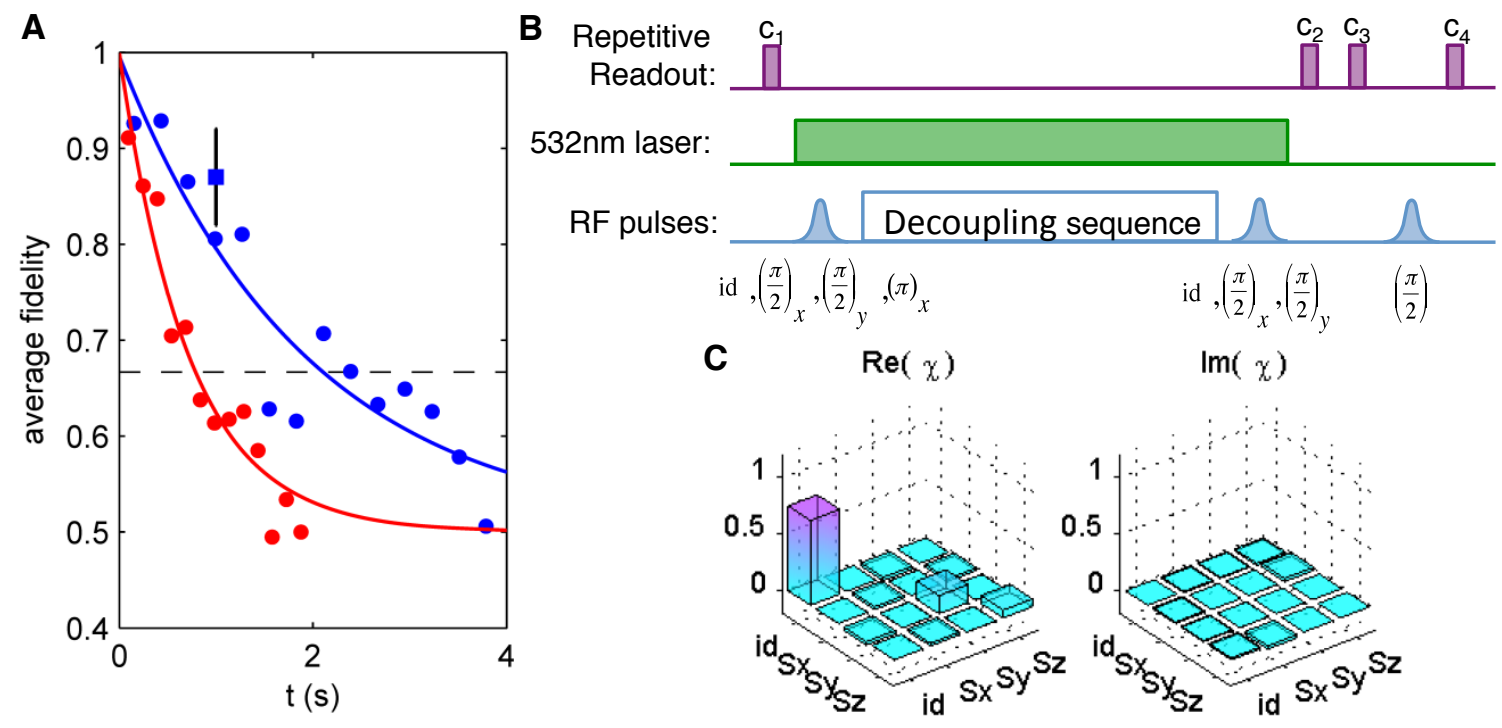